\title{
Uterine and tubal anatomical abnormalities in infertile women: diagnosis with routine hystero- salpingography prior to selective laparoscopy
}

\author{
M Heis, MD, FRCR, FFRRCSI, CST \\ Z Amarin, MD, FRCOG, FFPH \\ A Y Ibrahim, MD \\ N Obeidat, $M D$ \\ B Obeidat, MD, FRCOG \\ M Omari, MD \\ Jordan University of Science and Technology, Irbid, Jordan
}

Corresponding author: Z Amarin (zoamarin@hotmail.com)

\begin{abstract}
Objective. To assess the findings and usefulness of hysterosalpingography (HSG) as a routine investigation in the fertility workup prior to selective laparoscopy.

Design. Descriptive retrospective study.

Setting. A university hospital in the north of Jordan.

Subjects. All patients who underwent hysterosalpingography in the period 1 January - 31 December 2008.

Outcome measures. Detection of uterine and fallopian tube abnormalities and their correlation with laparoscopic findings.

Results. During the study period, 281 infertile women underwent HSG with no post-procedural complications. The mean (SD) age was 31.5 (5.9) years. Mean (SD) duration of infertility was 4 (3.4) years. Infertility was reported as primary and secondary in $119(42.3 \%)$ and $162(57.6 \%)$, respectively. Altogether, 281 patients and 562 tubes were examined. Of those, 402 were patent and 160 occluded. In only one woman were peritubal adhesions diagnosed. Because of hysterosalpingographically diagnosed tubal occlusion, 46 women (16.4\%) were referred for laparoscopy. Eight (17.3\%) of them were treated with unilateral salpingectomy, and 28 (60.8\%) with bilateral salpingectomy. Salpingolysis was performed on 7 (15.2\%) women; 3 (6.7\%) women had untreatable adhesions. The concordance was $71.7 \%$. The sensitivity of HSG was $80 \%$, the specificity $50 \%$, the negative predictive value $61 \%$, and the positive predictive value $71 \%$. Of the total of 281 women, 30 (10.7\%) conceived within 1 - 11 months after HSG.

Conclusion. The very high abnormal predictive value of HSG in the diagnosis of tubal occlusion suggests that this procedure could be performed as a screening examination.
\end{abstract}

\section{Introduction}

Infertility affects about $15 \%$ of the population, with at least 1 in 6 couples needing specialist help at some time in their lives because of infertility. ${ }^{1}$ After history taking, physical examination, semen analysis and ovulation studies, assessment of tubal patency is the next standard test. A universally agreed upon test for fallopian tube patency has not been established. A variety of investigation modalities are available that include hysterosalpingography (HSG), laparoscopic dye hydrotubation, hysterosalpingo contrast sonography (HyCoSy), selective salpingography, MR hysterosalpingography with an angiographic timeresolved 3D pulse sequence, radiography/MRI, 2D HyCoSy with contrast-tuned imaging, and falloposcopy.-5

Traditionally, HSG and laparoscopy with dye have been used in the diagnosis of tubal pathology. However, non-invasive methods are associated with false-positive results where occlusion is related to tubal spasm. Laparoscopy with dye is still considered to be the gold standard if tubal pathology is suspected, but it requires general anaesthesia and operating theatre facilities. Despite advanced technology and experience, complications during laparoscopy remain a major cause of significant morbidity and very seldom reveal any pathological conditions. ${ }^{6}$ Furthermore, infertility healthcare costs are difficult to calculate. There are few published data that determine the actual medical costs of adding an infertility evaluation test. ${ }^{7}$ Without such data, it is difficult to determine if a certain investigation would obviate the need to use other more invasive or more financially onerous tests.

The aim of this study was to assess the findings and define the role of HSG as a routine investigation in the fertility workup, prior to selective laparoscopy, in a tertiary referral institute in Jordan.

\section{Materials and methods}

Between 1 January and 31 December 2008, 281 pragmatic HSG studies were performed at the Jordan University of Science and Technology to investigate anatomical causes for subfertility on an outpatient basis during the proliferative phase of the menstrual cycle, prior to selective laparoscopy. All procedures were monitored fluoroscopically and interpreted by a radiologist. A water-soluble contrast medium (Omnipaque 33) was used. One photograph was taken when the cavity and fallopian tubes were filled, and one after overflow into the peritoneal cavity or when there was maximal filling without spillage. A late film was taken to detect contrast depots. Findings of tubal pathology were classified as normal, unilateral abnormality, bilateral abnormality, and findings suggestive of peritubal adhesions on the basis of loculation of contrast medium around the fallopian tube and restriction of flow away from the distal end. Proximal tubal occlusion was diagnosed by the 


\begin{tabular}{|ll|}
\hline \multicolumn{2}{|c|}{$\begin{array}{c}\text { Table I. Tubal pathology identified by } \\
\text { hysterosalpingography }\end{array}$} \\
\hline Bilateral hydrosalpinx & 27 \\
Unilateral hydrosalpinx & 19 \\
Bilateral distal occlusion & 5 \\
Unilateral distal occlusion & 27 \\
Bilateral proximal occlusion & 11 \\
Unilateral proximal occlusion & 16 \\
Bilateral combined proximal/distal occlusion & 6 \\
Unilateral combined proximal/distal occlusion & 5 \\
Periadnexal adhesions & 1 \\
\hline
\end{tabular}

\begin{tabular}{|lll|}
\hline \multicolumn{3}{|c|}{$\begin{array}{c}\text { Table II. Congenital uterine anomalies identified } \\
\text { by hysterosalpingography }\end{array}$} \\
\hline Attribute & Frequency & $\%$ \\
\hline No congenital anomalies & 258 & 91.8 \\
Bicornuate unicollis uterus & 9 & 3.2 \\
Arcuate uterus & 11 & 3.9 \\
Uterine septum & 1 & 0.4 \\
Hypoplastic uterine cavity & 2 & 0.7 \\
Total & 281 & 100 \\
\hline
\end{tabular}

absence of contrast medium beyond the isthmus, while the distal tubal occlusion was diagnosed on the basis of contrast medium within the ampulla but not passing through to the peritoneal cavity.

\section{Results}

The study population comprised 281 infertile women. Overall, the mean age (SD) was 31.5 (5.9) years, with a range of 18 - 46 years. Mean (SD) duration of infertility was 4 (3.4) years, range $1-21$ years. Infertility was reported as primary and secondary by $119(42.3 \%)$ and $162(57.6 \%)$, respectively.

Altogether, 281 patients and 562 tubes were examined. None of the women had only one tube. Of the investigated tubes, 402 were

\begin{tabular}{|c|c|c|}
\hline \multicolumn{3}{|c|}{$\begin{array}{l}\text { Table III. Uterine pathology identified by } \\
\text { hysterosalpingography }\end{array}$} \\
\hline Attribute & Frequency & $\%$ \\
\hline Normal opacification & 216 & 76.8 \\
\hline Congenital uterine anomalies & 23 & 8.1 \\
\hline Retroverted uterus with normal opacification & 14 & $5, .0$ \\
\hline Uterine fibroid & 15 & 5.3 \\
\hline Poor opacification with irregular outline & 4 & 1.4 \\
\hline Caesarean section scar & 4 & 1.4 \\
\hline Adhesions & 1 & 0.4 \\
\hline Irregular outline of uterine cavity & 4 & 1.4 \\
\hline Total & 281 & 100 \\
\hline
\end{tabular}

patent and 116 occluded. Table I shows tubal pathology, and Tables II and III show the congenital uterine anomalies and uterine pathology, respectively, as estimated by HSG. In all the 281 HSG investigations in this study, the procedure was tolerated well, with no post-procedural complications to warrant termination of the procedure, and no febrile morbidity was noted.

Because of hysterosalpingographically diagnosed tubal occlusion, 46 women (16.4\%) were referred for laparoscopy. Laparoscopy confirmed the findings shown on HSG. Eight (17.3\%) subjects underwent unilateral salpingectomy and 27 (58.6\%) bilateral salpingectomy. Salpingolysis was performed on 7 (15.2\%) women;4 (8.6\%) women had untreatable adhesions. The concordance was $71.7 \%$. The sensitivity of HSG was $80 \%$, specificity $50 \%$, negative predictive value $61 \%$, and positive predictive value $71 \%$ (see Table IV). The remaining 34 women were either lost to follow-up or were referred for in vitro fertilisation. Of the total of 281 women, $30(10.7 \%)$ conceived within 1 - 11 months after HSG.

\section{Discussion}

The issue of a gold standard is important. Laparoscopy and dye test is commonly used in most clinical studies on tubal factor subfertility as the reference standard. Some studies questioned the choice of laparoscopy and dye test as a gold standard procedure. Meta-analyses

\begin{tabular}{|llllll|}
\hline \multicolumn{5}{|c|}{ Table IV. Tubal pathology identified by hysterosalpingography and findings at laparoscopy } \\
\hline Tubal pathology identified by hysterosalpingography & \multicolumn{5}{l}{ Findings at laparoscopy } \\
\hline Type of pathology & $N$ & Normal & $\begin{array}{l}\text { One-sided } \\
\text { tubal occlusion }\end{array}$ & $\begin{array}{l}\text { Two-sided } \\
\text { tubal occlusion }\end{array}$ & $\begin{array}{l}\text { Peri-adnexal } \\
\text { adhesions }\end{array}$ \\
\hline Bilateral hydrosalpinx & & & 1 & 9 & 1 \\
Unilateral hydrosalpinx & 12 & 1 & 6 & 2 \\
Bilateral distal occlusion & 8 & 0 & 6 & 2 & 0 \\
Unilateral distal occlusion & 2 & 0 & 0 & 0 & 2 \\
Bilateral proximal occlusion & 9 & 1 & 6 & 3 & 0 \\
Unilateral proximal occlusion & 3 & 0 & 0 & 0 & 0 \\
Bilateral combined proximal/distal occlusion & 6 & 4 & 2 & 2 & 0 \\
Unilateral combined proximal/distal occlusion & 2 & 0 & 0 & 0 & 1 \\
Periadnexal adhesions & 3 & 0 & 2 & 0 & 1 \\
Total & 1 & 0 & 0 & 16 & 7 \\
\hline
\end{tabular}


comparing results of HSG and laparoscopy and dye test for the diagnosis of tubal pathology demonstrated that over one-third of the tubes found to be occluded at laparoscopy and dye test showed patency at HSG. ${ }^{8}$ Therefore, it could be said that the diagnosis of tubal occlusion can not be made with absolute certainty unless it is checked and probably confirmed by HSG, unless it is argued that the actual procedure of one or the other procedure was instrumental in affecting tubal patency, owing to the actual hydrostatic pressure exerted on the tubes during the procedure. In the current study, all 46 women (16.4\%) with hysterosalpingographically diagnosed tubal occlusion were confirmed by laparoscopy, confirming the value of HSG for intra-tubal pathology. In contrast, laparoscopy and dye test as a primary procedure has not proved to be a gold standard test, as some patients diagnosed with bilateral tubal occlusion by this technique were reported to have a 3-year cumulative pregnancy of $2 \% .{ }^{9}$ Fertiloscopy has been recently advocated as the procedure of choice for evaluation of tubal status, but further evaluation of its merits is necessary. ${ }^{10}$

The routine use of HSG in the fertility workup should be undertaken against the background of the possibility of faulty technique and artifacts. Hofmann et al. ${ }^{11}$ found that $17 \%$ of the films were technically inadequate. Artifacts include faulty insertion of the cannula, vaginal reflux, different tubal muscle tone and cornual spasm..$^{12,13}$

Although HSG has traditionally been used as a first-line technique for the diagnosis of tubal pathology, it is associated with false-positive results related to tubal spasm. In addition, the diagnostic accuracy of HSG could be influenced by lack of reproducibility. The interpretation of HSG results could be biased owing to variability within and between observers, especially regarding the interpretation of the possibility of adhesions. ${ }^{14}$ Furthermore, it has been estimated that clinicians were more reliable in diagnosing hydrosalpinx and tubal obstruction, while radiologists were more reliable in the detection of salpingitis isthmica nodosa and uterine adhesions. ${ }^{15}$

Some studies advocate a 3- to 6-month interval to allow for the so-called positive perturbation effect after normal HSG. Only patients who did not conceive during this interval were referred for laparoscopy with dye. ${ }^{16,17}$ In our study, of the total of 281 women, 30 (10.7\%) conceived within 1 - 11 months after HSG.
We conclude that the very high abnormal predictive value of HSG in the diagnosis of tubal pathology suggests that this procedure could be performed as a screening examination, whereas diagnostic laparoscopy could be used as a second-line technique.

1. Hull MG, Glazener CM, Kelly NJ, et al. Population study of causes, treatment, and outcome of infertility. BMJ 1985;291:1693-1697.

2. Ahinko-Hakamaa KM, Huhtala H, Tinkanen H. Confirmation of tubal patency in hysterosalpingo-contrast sonography by transvaginal hydrolaparoscopy. Acta Obstet Gynecol Scand 2009;88(3):286-290.

3. Sadowski EA, Ochsner JE, Riherd JM, et al. MR hysterosalpingography with an angiographic timeresolved 3D pulse sequence: assessment of tubal patency. Am J Roentgenol 2008;191(5):1381-1385.

4. Freeman-Walsh CB, Fahrig R, Ganguly A, Rieke V, Daniel BL. A hybrid radiography/MRI system for combining hysterosalpingography and MRI in infertility patients: initial experience. Am J Roentgenol 2008;190(2):W157-160.

5. Lanzani C, Savasi V, Leone FP, Ratti M, Ferrazzi E. Two-dimensional HyCoSy with contrast tuned imaging technology and a second-generation contrast media for the assessment of tubal patency in an infertility program. Fertil Steril 2008; 29 Sep [Epub ahead of print].

6. Tarik A, Fehmi C. Complications of gynaecological laparoscopy: a retrospective analysis of 3572 cases from a single institute. J Obstet Gynaecol 2004;24:813-816.

7. Stovall DW, Allen BD, Sparks AE, Syrop CH, Saunders RG, VanVoorhis BJ. The cost of infertility evalution and therapy: findings of a self-insured university healthcare plan. Fertil Steril 1999;72(5):778-784.

8. Swart P, Mol BW, van der Veen F, van Beurden M, Redekop WK, Bossuyt PM. The accuracy of hysterosalpingography in the diagnosis of tubal pathology: a meta-analysis. Fertil Steril 1995;64(3):486-491.

9. Mol BW, Collins JA, Burrows EA, van der Veen F, Bossuyt PM. Comparison of hysterosalpingography and laparoscopy in predicting fertility outcome. Hum Reprod 1999;14(5):1237-1242.

10. Watrelot A, Nisolle M, Chelli H, Hocke C, Rongieres C, Racinet C. Is laparoscopy still the gold standard in infertility assessment? A comparison of fertiloscopy versus laparoscopy in infertility. Results of an international multicentre prospective trial: the 'FLY' (Fertiloscopy-Laparoscopy) study. Hum Reprod 2003;18(4):834-839.

11. Hofmann GE, Scott RT, Rosenwaks Z. Common technical errors in hysterosalpingography. Int J Fertil 1992;37(1):41-43.

12. Shah SM, Towobola OA, Masihleho M. Diagnosis of fallopian tube patency. East Afr Med J 2005;82(9):457-462.

13. Cheong YC, Li TC. Evidence-based management of tubal disease and infertility. Current Obstetrics \& Gynaecology 2005;15(5):306-313.

14. Glatstein IZ, Sleeper LA, Lavy Y, et al. Observer variability in the diagnosis and management of the hysterosalpingogram. Fertil Steril 1997;67(2):233-237.

15. Renbaum L, Ufberg D, Sammel M, Zhou L, Jabara S, Barnhart K. Reliability of clinicians versus radiologists for detecting abnormalities on hysterosalpingogram films. Fertil Steril 2002;78(3):614-618.

16. Perquin DA, Beersma MF, de Craen AJ, Helmerhorst FM. The value of Chlamydia trachomatis-specific IgG antibody testing and hysterosalpingography for predicting tubal pathology and occurrence of pregnancy. Fertil Steril 2007;88(1):224-226.

17. Evers JL, Land JA, Mol BW. Evidence-based medicine for diagnostic questions. Semin Reprod Med 2003;21(1):9-15 